\title{
A STEADY PSEUDO-COMPRESSIBILITY APPROACH BASED ON UNSTRUCTURED HYBRID FINITE VOLUME TECHNIQUES APPLIED TO TURBULENT PREMIXED FLAME PROPAGATION
}

\author{
W. M. C. Dourado, \\ A-P - IAE - CTA \\ ABSTRACT \\ São José dos Campos - 11228-904 \\ $\mathrm{SP}-$ Brazil \\ A pseudo-compressibility method for zero Mach number turbulent reactive \\ flows with heat release is combined with an unstructured finite volume \\ hybrid grid scheme. The spatial discretization is based on an overlapped cell \\ vertex approach. An infinite freely planar flame propagating into a turbulent \\ medium of premixed reactants is considered as a test case. The recourse to a \\ flamelet combustion modeling for which the reaction rate is quenched in a \\ continuous way ensures the uniqueness of the turbulent flame propagation \\ velocity. To integrate the final form of discretized governing equations, a \\ three-stage hybrid time-stepping scheme is used and artificial dissipation \\ terms are added to stabilize the convergence path towards the final steady \\ solution. The results obtained with such a numerical procedure prove to be \\ in good agreement with those reported in the literature on the very same \\ flow geometry. Indeed, the flame structure as well as its propagation \\ velocity are accurately predicted thus confirming the validity of the \\ approach followed and demonstrating that such a numerical procedure will \\ be a valuable tool to deal with complex reactive flow geometries. \\ J. L. F. Azevedo,
} \author{
ASE-N - IAE - CTA \\ São José dos Campos - 11228-904
}

$\mathrm{SP}-$ Brazil

Azevedo@iae.cta.br

\section{INTRODUCTION}

Steady and unsteady zero Mach number reactive flows in the sense of Majda and Sethian (1985), are often encountered in many systems of practical interest such as furnaces or jet engines. In such cases, the pressure can be considered as being thermodynamically constant e.g. the density variations are uniquely related to the temperature changes due to the heat released by the combustion process. When compressible flow solvers are employed in such situations, their convergence rate is becoming so slow that specific procedures such as preconditioning techniques have to be employed to improve them, but in the limit $M a \rightarrow O$ even the recourse to such techniques is not sufficient to ensure a satisfactory convergence rate (Dourado and Azevedo, 1996 and 1999; Dourado et al., 2000). Accordingly, specific numerical strategies have to be employed such as the Simple, the PISO or the pseudocompressibility technique (Chorin, 1967). The latter approach presents the immense advantage over the two others that its introduction in a compressible flow solver is easy and leaves the structure of the computer program largely unmodified while allowing the calculations of both inert and reacting flows (Corvellec, 1998; Corvellec et al.; 1999). On the other hand, during the last two decades, the finite volume unstructured grid technique has shown its ability to cope with a wide range of complex compressible flow geometries (Jameson et al., 1986, Barth and Jespersen, 1989) including the use of mesh refinement techniques (Batina, 1989, Trépanier et al., 1991, Nomura and Hughes, 1992) that are appealing for properly resolving the high spatial gradients that are potentially present in the flows under investigation. Thus, the temptation is strong to combine the advantages of both the pseudo-compressibility method and the finite volume unstructured grid technique to develop a computer code able to deal with low Mach number inert or reacting flows. Along these lines, the present work is devoted to the development and the test of such a combination that results in the development of a 2-D computer code that will be tested on the geometry of an 1-D freely propagating turbulent premixed flame whose velocity is known, thanks to the formulation retained for the combustion modeling. 


\section{PHYSICAL MODELING AND NUMERICAL STRATEGY}

\section{Combustion Model}

Since the present study is mainly concerned with the numerical method of solution of the governing equations rather than the modeling of the combustion process itself, only a brief description of the modeling is given here. The formulation retained is similar to that of Corvellec et al. (1999). This choice is motivated by the fact that such a modeling yields a turbulent premixed flame that propagates at a velocity that can be directly derived from the so-called KPP analysis (Kolmogorov et al., 1937, Corvellec et al., 1999). Consequently, such a test case permits an unambiguous evaluation of the capability of the code to predict the correct turbulent flame speed which is intrinsic to the set of equations considered. So, the recourse to the comparison with experimental data is not required at this stage as long as it is not the physical modeling itself which is to be tested. A simplified Bray-Moss-Libby like formulation (Bray and Moss, 1977, Bray et al., 1984, Bray et al., 1988), thereafter referred to as BML is used with a quenched mean reaction rate. Accordingly, the isenthalpic combustion process takes place in infinitely thin-laminar like reactive interfaces (flamelets) whose thickness is less than the Kolmogorov scale and whose laminar flame velocity is negligible compared to the velocity fluctuations associated with turbulence. An observer recording the temperature signal at a given point within the flow would obtain basically two values of the temperature signal e.g. $T=T_{b}$ in the fully burnt products and $T=T_{r}$ in the unburned mixture. The combustion process can then be represented through the evolution of a single bi-valued progress variable $c$ ( $c=0$ in the unburned mixture and to $c=1$ in the fully burnt products) which plays the role of a reduced temperature via the following relation:

$$
c=\frac{T-T_{r}}{T-T_{b}}
$$

The zero Mach number hypothesis leads to the following equation of state:

$$
\rho T=\rho_{r} T_{r}=\rho_{b} T_{b}
$$

or alternatively :

$$
\rho=\frac{\rho_{r}}{1+\chi c}
$$

where the heat release parameter is defined by:

$$
\chi=\frac{T_{b}-T_{r}}{T_{r}}
$$

With use of the classical Favre average (Favre, 1965), the governing equation for the mean progress variable $\tilde{c}=\frac{\overline{\rho c}}{\bar{\rho}}$ can be written as:

$$
L(\bar{\rho} \widetilde{c})=\bar{w}
$$

where the operator $L$ regroups the convective and diffusive operators. To ensure the existence of a unique propagation velocity for the turbulent flame brush, a continuous quenched form for the mean reaction rate $\bar{w}$ is chosen (Corvellec, 1998; Corvellec et al., 1999), namely: $\bar{w}= \begin{cases}0 & \tilde{c} \leq \chi c^{\star} \\ C_{w}(1+\chi) \frac{\left(\tilde{c}-\chi c^{\star}\right)(1-\tilde{c})}{(1+\chi \tilde{c})^{2}} & \text { otherwise }\end{cases}$

\section{Pseudo-Compressibility Technique}

The pseudo or artificial compressibility technique was proposed initially by Chorin (1967) to deal with inert incompressible flows. It has been extended to steady reactive flows by Bruel et al. (1996), and to unsteady reactive flows by Corvellec et al. (1999). The basic idea behind this technique consists in modifying the continuity equation to introduce a finite sound speed during the course of the convergence process toward the solution. Thus, the continuity equation permits de facto the calculation of the static pressure and reads as:

$\frac{1}{\beta} \frac{\partial \bar{p}}{\partial \tau}+\frac{\partial \bar{\rho} \tilde{u}_{i}}{\partial x_{i}}=0$

where $\beta$ is the pseudo-compressibility factor expressed in $\mathrm{m}^{2} / \mathrm{s}^{2}$ and $\tau$ is the pseudo-time. This equation is physically meaningful when convergence is reached in $\tau$ e.g. when $\frac{\partial \bar{p}}{\partial \tau}$ vanishes. The pseudo-sound speed is given by:

$$
a=\sqrt{u^{2}+\beta}
$$

and the local corresponding pseudo-Mach number $\mathrm{Ma}^{*}$ defined by $M a^{*}=\frac{u}{a}$ is thus always less than one as soon as $\beta$ is larger than zero. For a 1-D turbulent flame and using an implicit structured finite differences approach, Bruel et al. (1996) have shown that i) the value of the pseudocompressibility factor $\beta$ influences strongly the convergence rate of the calculations and ii) a value $\beta \approx 5 u_{r e f}^{2}$ to $10 u_{r e f}^{2}$ where $u_{r e f}$ is the largest convective velocity expected in the flow is found to be a good compromise in terms of convergence rate.

\section{Mean Governing Equations}

In spite of the fact that the test case considered here is one-dimensional, the governing equations are cast into a two-dimensional form since an unstructured approach for dealing with such equations cannot be formulated otherwise. For the simplified combustion model retained here, the main mean variables that describe the turbulent reactive flow are the density $\bar{\rho}$, the Cartesian velocity components $\tilde{u}_{i}$, the static pressure $\bar{p}$ and the progress reaction variable $\tilde{c}$. The steady governing equations that include the pseudo-compressibility approach are given by:

$$
\frac{\partial q}{\partial \tau}+\frac{\partial E_{e_{i}}}{\partial x_{i}}-\frac{\partial E_{v_{i}}}{\partial x_{i}}=S
$$


with $x_{1} \equiv x$ and $x_{1} \equiv y$, the various vectors can be expressed as:

$q=\left\{\begin{array}{c}\bar{p} \\ \bar{\rho} \tilde{\mathrm{u}} \\ \bar{\rho} \tilde{v} \\ \bar{\rho} \tilde{c}\end{array}\right\} \quad E_{e_{x}}=\left\{\begin{array}{c}\beta \bar{\rho} \tilde{\mathrm{u}} \\ \bar{\rho} \tilde{u}^{2}+\bar{p} \\ \bar{\rho} \tilde{\mathrm{u}} \tilde{v} \\ \bar{\rho} \tilde{\mathrm{u}} \tilde{c}\end{array}\right\} \quad E_{e_{y}}=\left\{\begin{array}{c}\beta \bar{\rho} \tilde{v} \\ \bar{\rho} \tilde{v} \\ \bar{\rho} \tilde{v}^{2}+\bar{p} \\ \bar{\rho} \tilde{v} \tilde{c}\end{array}\right\}$

$E_{v_{x}}=\left\{\begin{array}{c}0 \\ \overline{\sigma_{x x}}-\widetilde{\rho} \widetilde{u^{\prime \prime} u^{\prime \prime}} \\ \overline{\sigma_{x y}}-\widetilde{\rho} \widetilde{u^{\prime \prime} v^{\prime \prime}} \\ \overline{\rho D \frac{\partial c}{\partial x}}-\widetilde{\rho u^{\prime \prime} c^{\prime \prime}}\end{array}\right\} \quad E_{v_{y}}=\left\{\begin{array}{c}0 \\ \overline{\sigma_{x y}}-\bar{\rho} \widetilde{u^{\prime \prime} v^{\prime \prime}} \\ \overline{\sigma_{y y}}-\bar{\rho} \widetilde{v^{\prime \prime} v^{\prime \prime}} \\ \frac{\rho D \frac{\partial c}{\partial y}}{-\rho} \widetilde{u^{\prime \prime} c^{\prime \prime}}\end{array}\right\}$

$S=\left\{\begin{array}{c}0 \\ 0 \\ 0 \\ \bar{w}\end{array}\right\}$

where $\bar{w}$ is the mean reaction rate given by Eq. (6) and $\sigma_{i j}$ is the laminar stress tensor expressed for a Newtonian fluid by:

$\sigma_{i j}=\mu_{l}\left(\frac{\partial u_{i}}{\partial x_{j}}+\frac{\partial u_{j}}{\partial x_{i}}-\frac{2}{3} \frac{\partial u_{k}}{\partial x_{k}} \delta_{i j}\right)$

In Eq. (10), $D=\frac{v_{l}}{S c_{l}}$ is the laminar diffusion coefficient, $v_{l}$ the kinematic laminar viscosity and $S c_{l}$ the laminar Schmidt number. The Reynolds stresses, present in Eq. (10) are closed with a Boussinesq-like expression, namely (Frisch, 1995):

$\widetilde{\rho} \widetilde{u_{i}^{\prime \prime} u_{j}^{\prime \prime}}=-\mu_{t}\left(\frac{\partial \tilde{\mathbf{u}}_{i}}{\partial x_{j}}+\frac{\partial \tilde{\mathbf{u}}_{j}}{\partial x_{i}}\right)+\frac{2}{3}\left(\bar{\rho} \tilde{k}+\mu_{t} \frac{\partial \tilde{\mathbf{u}}_{k}}{\partial x_{k}}\right)$

Where $\tilde{k}=\frac{1}{2} \frac{\overline{\rho u_{i}^{\prime \prime} u_{j}^{\prime \prime}}}{\bar{\rho}}$ is the turbulence kinetic energy. The turbulent viscosity coefficient can be expressed as a function of $\widetilde{k}$ and of its dissipation rate $\widetilde{\varepsilon}$ by (Launder and Spalding, 1974):

$\mu_{t}=\bar{\rho} C_{\mu} \frac{\tilde{k}^{2}}{\tilde{\epsilon}}$

with $C_{\mu}$ being equal to 0.09 .

In the present work, the turbulence field is supposed to be frozen at the level that prevails in the fresh reactants so that $\widetilde{k}=\widetilde{k}_{r}$ and $\widetilde{\varepsilon}=\widetilde{\varepsilon}_{r}$ with: $\tilde{k}_{r}=\frac{3}{2} \overline{\mathrm{u}_{r}^{\prime 2}} \quad \tilde{\epsilon}_{r}=C_{\mu}^{0.75} \frac{\tilde{k}_{r}^{3 / 2}}{l_{i}}$

where $u_{r}^{\prime}=\sqrt{\overline{u_{r}^{\prime 2}}}$ and $l_{i}$, to be prescribed, stand for the rms velocity fluctuations and the turbulence integral length that prevail in the fresh reactants, respectively.

The turbulent mass flux present in Eq. (10) is also expressed through a gradient approximation, namely (Bray et al., 1984):

$\bar{\rho} \widetilde{u_{i}^{\prime \prime} c^{\prime \prime}}=-\bar{\rho} D_{t} \frac{\partial \tilde{c}}{\partial x_{i}}$

where $D_{t}=\frac{v_{t}}{S c_{t}}$ is the turbulent diffusion coefficient, $v_{t}=\frac{\mu_{t}}{\bar{\rho}}$ the turbulent kinematic viscosity and $\overrightarrow{\mathrm{n}}$ the turbulent Schmidt number. The closed form of Eqs. (9) is now written in an integral form using the Green's theorem:

$V \frac{\partial}{\partial \tau} \int_{V} q d V+\oint_{\mathbf{S}} \mathbf{E}_{\mathbf{e}} \cdot \overrightarrow{\mathbf{n}} d \mathbf{S}=\oint_{\mathbf{S}} \mathbf{E}_{\mathbf{v}} \cdot \overrightarrow{\mathbf{n}} d \mathbf{S}+\int_{V} \mathbf{S} d V$

where $\vec{n}$ represents the outgoing normal unitary vector to surface $S$ of volume $V$ and $\mathrm{E}_{\mathrm{e}}$ and $\mathrm{E}_{\mathrm{v}} \$$ are the convective and diffusion flux vectors whose components are given in Eq. (10). In two dimensional Cartesian notation, the product $\vec{n} d S$ is equal to $\Delta y \vec{i}-\Delta x \vec{j}$ where $\vec{i}$ and $\vec{j}$ are the basis vectors.

\section{Spatial Discretization}

As the control volume can be constructed a priori with different type of elements (triangles and/or quadrangles) on hybrid grid topologies, the elementary cell of the grid considered here is simply called an element. Thus, the control volume $V$ is defined by the external boundary surface of all elements sharing a common node, as sketched in Fig. (1). The spatial discretization adopted here is based on a cell center finite volume method adapted for cell vertex hybrid elements (Jameson et al., 1986). This scheme proved to be efficient for dealing with steady and unsteady inert flows as reported by Mavriplis et al. (1989), Mavriplis and Venkatakrishnan (1995), Dourado and Azevedo (1996), Mavriplis (1998), Dourado and Azevedo (1999) and Dourado et al. (2000). The discretized form of Eq. (16) is written as:

$V \frac{\partial \bar{q}_{i}}{\partial \tau}+\sum_{f=1}^{n f}\left(\bar{E}_{e_{x} f} \Delta y-\bar{E}_{e_{y} f} \Delta x\right)=\sum_{f=1}^{n f}\left(\bar{E}_{v_{x} f} \Delta y-\bar{E}_{v_{y} f} \Delta x\right)+V \bar{S}$

where

$\bar{q}=\frac{1}{V} \int_{V} \mathbf{q} d V$

is the average main variables vector, and 


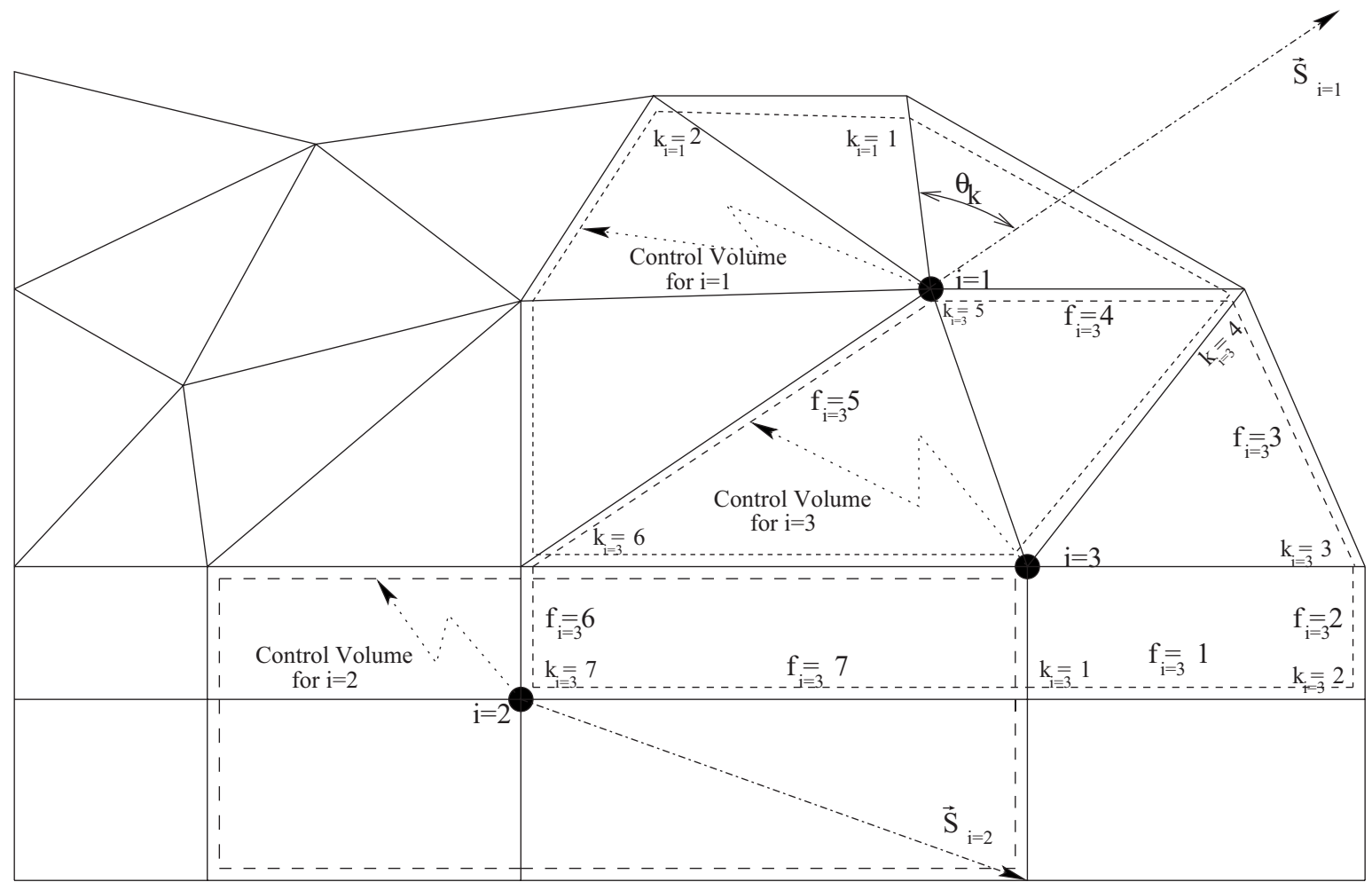

Figure 1 - Domain of influence of nodes 1, 2 and 3 and its local stretching for hybrid unstructured grid.

$\bar{S}=\frac{1}{V} \int_{V} \mathbf{S} d V$

is the average source term vector. $\bar{E}$ is the average flux vector at face $f$ calculated as the arithmetic average of the two fluxes located on nodes which delimit this face. Further details on triangular and hybrid elements in unstructured grids are presented in the studies that have been referred to above.

\section{Artificial Dissipation}

For high Reynolds number flows, it is frequently required to stabilize the calculations by adding explicitly some artificial dissipation to damp out numerical oscillations. These additional dissipative terms must be kept at a level that does not endanger the accuracy of the numerical solution. Accordingly, and as suggested by Dourado et al. (2000), the dissipation terms introduced in the systems of equations are written as:

$D_{a}\left(q_{i}\right)=\sum_{k=1}^{n n}\left[\Delta_{k}-\Delta_{i}\right]\left[\frac{A_{k}+A_{i}}{2}\right]$

where

$\Delta(q)=-k^{(4)} \nabla^{2} q$

and $n n$ is the number of nodes located on the boundary of the control volume $i$, as it is sketched in Fig. (1).

The constant $k^{(4)}$ which defines the level of the fourth order dissipation term is taken equal to $1 / 256$ for all the calculations presented in this work and the coefficient $A$ is adjusted according to the grid stretching by:

$A_{k}=\alpha_{1 k} \cos ^{2} \theta_{\mathrm{k}}+\alpha_{2 k} \sin ^{2} \theta_{\mathrm{k}}$

Subscripts $i$ and $k$ refer to the variable evaluation at nodes $i$ and $k$, respectively. At each mesh point, the coefficients $\alpha_{1}$ and $\alpha_{2}$ of Eq. (22) are calculated with the maximum eigenvalue $\lambda$ associated with the convective terms by:

$\alpha_{1}=\phi(s) \frac{1}{s+1} \lambda \quad \alpha_{2}=\phi\left(s^{-1}\right) \frac{s}{s+1} \lambda$

where $s$ represents the magnitude of the stretching vector $\vec{S}$ (see Fig. (1)) and $\phi(r)=1+r^{2 / 3}$ For an unstructured mesh, the maximum eigenvalue $\lambda$ at each mesh point is evaluated by the following discrete approximation:

$\lambda=\sum_{f=1}^{n f}\left|\bar{u}_{f} \Delta \mathrm{y}_{f}-\bar{v}_{f} \Delta \mathrm{x}_{f}\right|+\bar{a}_{f} \sqrt{\Delta \mathrm{x}^{2}+\Delta \mathrm{y}^{2}}$

where $\bar{u}, \bar{v}$ and $\bar{a}$ are the average values of the velocity components and of the sound speed calculated from the two nodes which delimit the face $f$ and cumulated over all the boundary faces of the control volume considered. Finally, since the code is edge based database organized, the artificial dissipation term formulation was enhanced in order to be usable in the context of hybrid grids where triangular and quadrilateral elements coexist. One should observe that, although quadrilateral meshes were used in the present simulations, the code treats all grids in a fully 
unstructured way. The reader should be aware that the definition of an unstructured grid is not necessary related to the shape of the control volumes but to the form in which these control volumes are addressed in the code.

\section{Pseudo-time marching}

As exposed before, when one has recourse to the pseudo-compressibility method to solve the flow governing equations, the solution is physically meaningful only when a steady state in pseudo-time is reached, so, even to seek steady state solutions, a time-stepping procedure has to be used. Thus, the discretized equations system (17) is first written as:

$V_{i} \frac{d q_{i}}{d \tau}+\left[C\left(q_{i}\right)-D v\left(q_{i}\right)-D_{a}\left(q_{i}\right)\right]=S\left(q_{i}\right) \quad i=1 \ldots n$

where the residuals of the convective, diffusive and stabilizing terms are denoted by $C(q) D_{v}(q)$ and $D_{a}$, respectively, and the source term is represented by $S(q)$.
Then, a Runge-Kutta explicit three-stage hybrid timestepping scheme is adopted due to its satisfactory performance, simplicity and low computational cost. The scheme proposed by Manzari et al. (1998), to advance the solution in pseudo-time is implemented here under the following form:

$q_{i}^{(0)}=q_{i}^{n}$

$q_{i}^{\kappa}=q_{i}^{(0)}-\alpha_{\kappa} \frac{\Delta \tau}{V_{i}}\left[C\left(q_{i}^{(\kappa-1)}\right)-D_{v}\left(q_{i}^{(\kappa-1)}\right)-S\left(q_{i}^{(\kappa-1)}\right)-D_{a}\left(q_{i}^{(\kappa-1)}\right)\right]$

$q_{i}^{n+1}=q_{i}^{(3)} \quad, \kappa=1,2,3$

The operators $C, D_{v}$ and $S$ are expressed by using the vector properties $q_{i}^{\left({ }^{(-1)}\right.}$ from stage $\kappa-1$, while the added artificial dissipation term is calculated only in the first stage and held constant throughout the next stages. The coefficients appearing in Eq. (26) are given the values $\alpha_{1}=0.6, \alpha_{2}=0.6$ and $\alpha_{3}=1.0$.

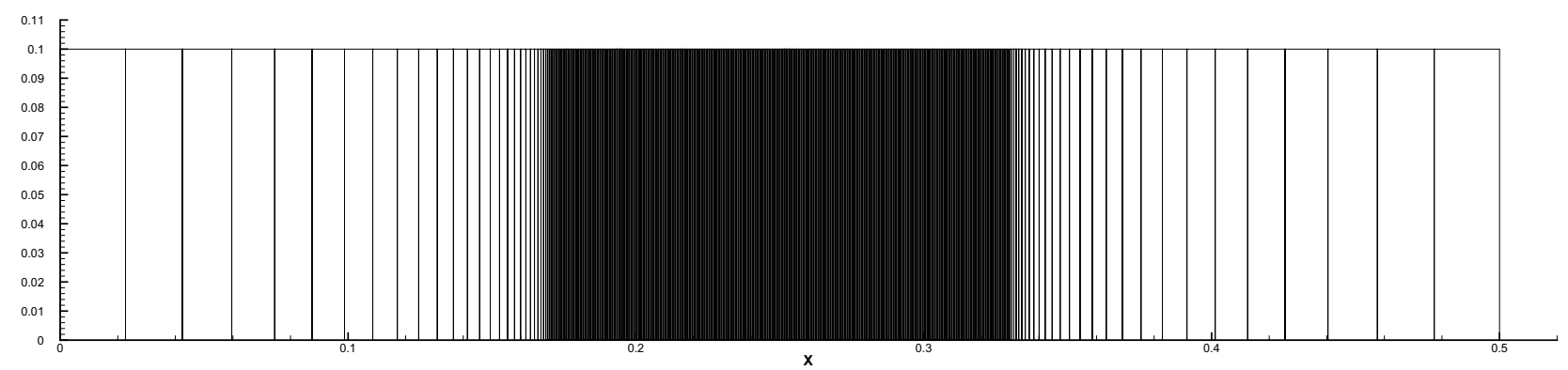

Figure 2 - Computational grid mesh 1 with 355 nodes and 354 quadrilateral elements.

\section{RESULTS}

The 1-D turbulent premixed flame referenced by Corvellec (1998) as Case I is considered here to test the present numerical approach. The parameters defining such a slow propagating flame are given in Table (1).

\begin{tabular}{|c|l|l|l|l|l|l|l|l|}
\hline$\chi$ & $\chi c^{*}$ & $\begin{array}{l}S_{t} \\
(\mathrm{~m} / \mathrm{s})\end{array}$ & $\begin{array}{l}\bar{u}_{r} \\
(\mathrm{~m} / \mathrm{s})\end{array}$ & $\begin{array}{l}u_{r}^{\prime} \\
(\mathrm{m} / \mathrm{s})\end{array}$ & $\begin{array}{l}l_{i} \\
(\mathrm{~m})\end{array}$ & $S c_{t}$ & $\begin{array}{l}C_{w} \\
\mathrm{~kg} / \\
\mathrm{m}^{3} / \mathrm{s}\end{array}$ & $\begin{array}{l}\rho_{r} \\
\mathrm{~kg} / \mathrm{m}^{3} / \\
\mathrm{s}\end{array}$ \\
\hline 5 & 0.03958 & 0.5 & 0.5 & 1.0 & $10^{-3}$ & 0.75 & 200 & 1.1886 \\
\hline
\end{tabular}

Table 1 - Parameters defining the 1-D turbulent premixed flame considered as test case.

The boundary condition imposed at the reactants side e.g. $\bar{u}_{r}=S_{t}$ and $\bar{c}=\widetilde{c}=0$ has been chosen so that the turbulent flame brush will be steady in the computational domain which consists of a strip of $0.5 \mathrm{~m}$ long and $0.1 \mathrm{~m}$ in height with only one division in the latter direction. An example of grid, called mesh 1 with 355 nodes in the longitudinal direction and 354 quadrilateral elements is presented in Fig. (2). In order to properly resolve the strong gradients that are to be expected in the turbulent brush near the quenching point, the mesh is highly refined in the region located between $0.17 m \leq x \leq 0.33 m$ with a minimum mesh spacing $\Delta x_{\min }$ equal to $5.33310^{-4} \mathrm{~m}$.

Due to the high Reynolds number flow hypothesis, the laminar diffusive transports will be neglected in front of their turbulent counterparts. To ensure 1-D like conditions on such a 2-D mesh, a symmetry condition is imposed at the lower and upper boundary lines parallel to the flow and are applied to the corresponding nodes and faces. Such a condition corresponds to the imposition of zero transverse velocity and gradients. At the burned products side, the pressure is imposed and all other variables are extrapolated. The initial condition for the progress variable $\widetilde{c}$ is given by a ramp form function that goes from $\widetilde{c}=0$ at $x=0.27 \mathrm{~m}$ to $\widetilde{c}=1$ at $x=0.32 \mathrm{~m}$. The Courant-Frederich-Lewis (CFL) number used in these calculations is equal to 0.94 and is kept constant throughout the computational domain. Accordingly, the pseudo time step $\Delta \tau$ is variable in the computational domain with a minimum value used in the region of greatest refinement. Such a CFL number is given by:

$C F L_{\tau}=\lambda_{b} \frac{\Delta \tau}{\Delta \mathrm{x}_{\min }}$ 


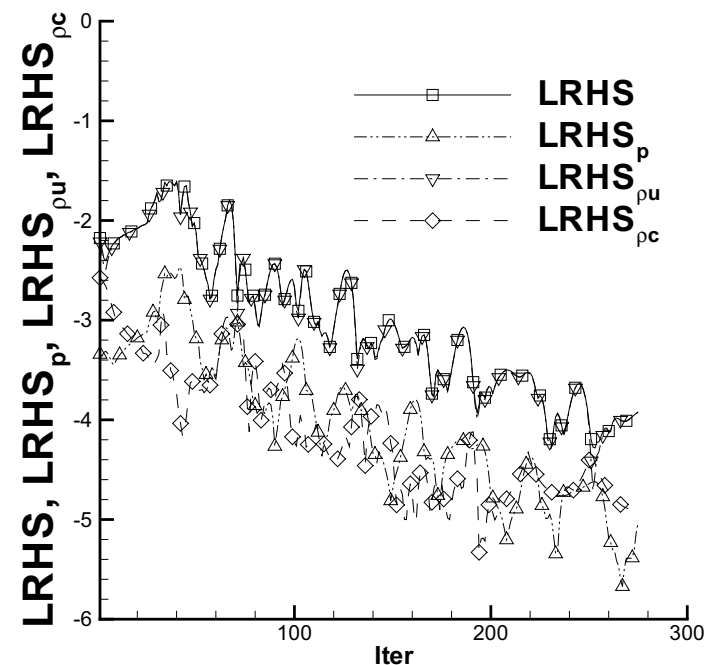

Figure 3 - Convergence history of residue using mesh 1 with $\beta=75 \mathrm{~m}^{2} / \mathrm{s}^{2}$.

where $\lambda_{b}=u_{b}+a_{b} \quad$ is the largest convective eigenvaluebased on the pseudo-sound speed given by Eq. (8). As a consequence, the minimum pseudo-time step for mesh 1 is equal to $2.10^{-5} \mathrm{~s}$. The convergence criterion set to $10^{-4}$ was met after 27500 iterations through a convergence history presented in Fig. (3). The corresponding profiles of pressure gradient, mean progress variable and mass flux obtained on mesh 1 are presented in Fig. (4).

The curvature at the foot of the flame brush is extremely strong and this illustrates why such a highly stretched mesh has to be used to capture properly the progress variable and pressure gradients in that zone. The profile of pressure gradient plotted against the progress reaction variable $\widetilde{c}$ is displayed in Fig. (5).

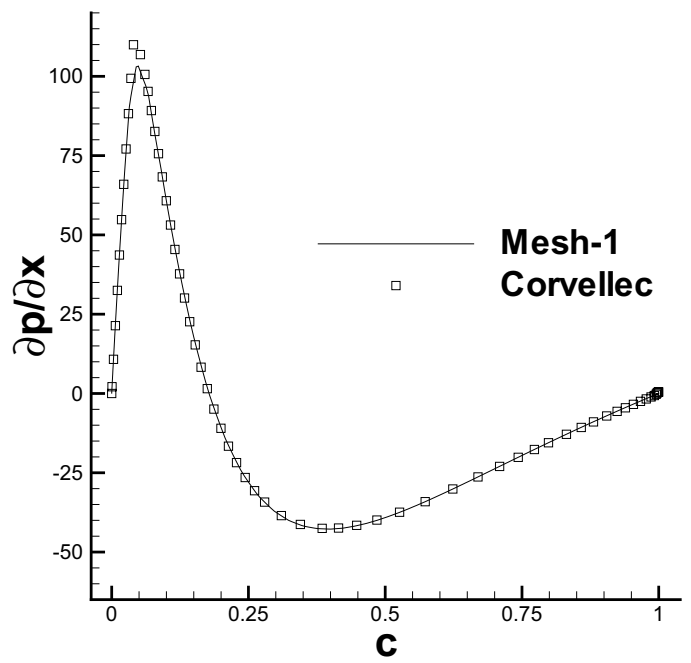

Figure 4 - Profiles of mean reaction progress variable, mean mass flux and pressure gradient versus $\mathrm{x}$ co-ordinate.

A good agreement is obtained between the present results and those of Corvellec (1998) with a difference in the maximum value of the pressure gradient of less than $6 \%$.

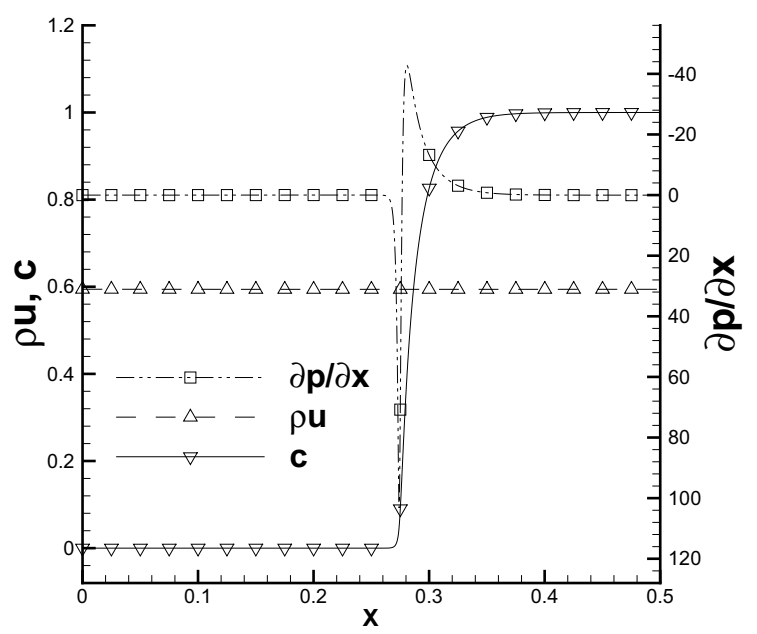

Figure 5 - Profiles of mean pressure gradient versus the mean reaction progress variable (mesh $1, \beta=75 \mathrm{~m}^{2} / \mathrm{s}^{2}$ ).

Consequently, the criterion convergence chosen here appears to be correctly adapted to get accurate converged results. To investigate the sensitivity of our results to the mesh refinement, three other meshes have been considered that differ from mesh 1 by their refinement: i) mesh 2 possesses $50 \%$ more divisions in the $x$-direction in the interval $0.17 m \leq x \leq 0.33 m$ with $\Delta x_{\min }=3.5610^{-4} \mathrm{~m}$; ii) mesh 3 has $100 \%$ more divisions in the $x$-direction on the same interval, which gives $\Delta x_{\min }=2.6710^{-4} \mathrm{~m}$ and iii) mesh 4 has the same number of nodes as mesh 3 but the interval of refinement is reduced to $0.22 m \leq x \leq 0.30 \mathrm{~m}$, which gives a minimum mesh discretization of $\Delta x_{\min }=1.3310^{-4} \mathrm{~m}$.

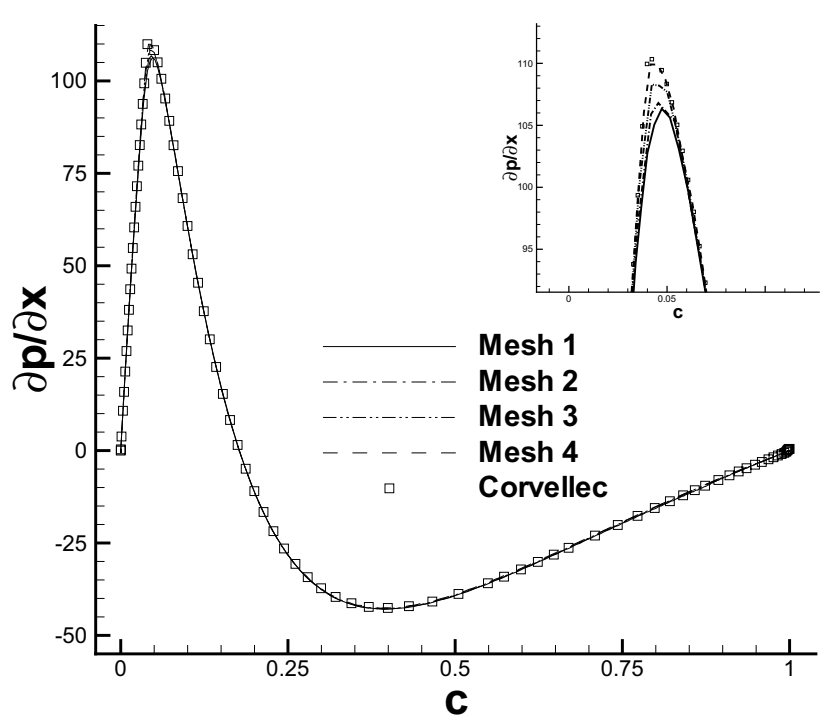

Figure 6 - Profiles of mean pressure gradient versus the mean reaction progress variable for meshes $1,2,3$ and 4 .

It should be remembered than in her study, Corvellec (1998) was using an auto-adaptive mesh refinement with a 
minimum spacing $\Delta x_{\min }$ equal to $7.110^{-5} \mathrm{~m}$. The results obtained with the different grids are presented in Fig. (6) where the profiles of the pressure gradient are plotted versus the mean reaction progress variable.

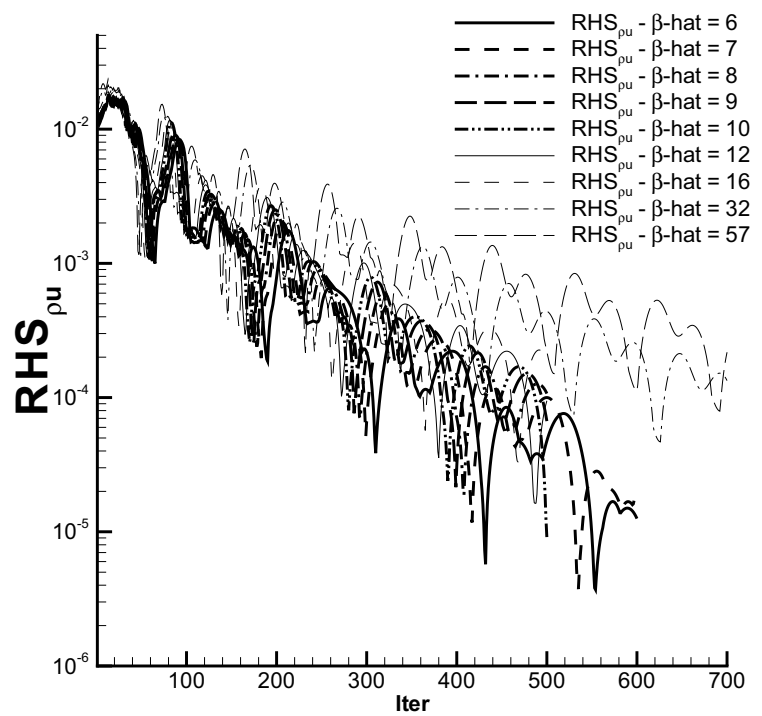

Figure 7 - Residue history for the $\mathrm{x}$-direction momentum equation for several values of $\hat{\beta}$.

It can be clearly seen that as soon as the mesh is sufficiently refined, no substantial difference can be observed with the results by Corvellec (1998), obtained, it should be stressed with a completely different numerical formulation. A short analysis of the influence of the choice of the pseudocompressibility factor $\hat{\beta}=\frac{\beta}{u_{b}^{2}}$ on the convergence rate was carried out here and Fig. (7) presents the convergence history of the residue of the momentum equation in the $x$ direction obtained for different values of $\hat{\beta}$.

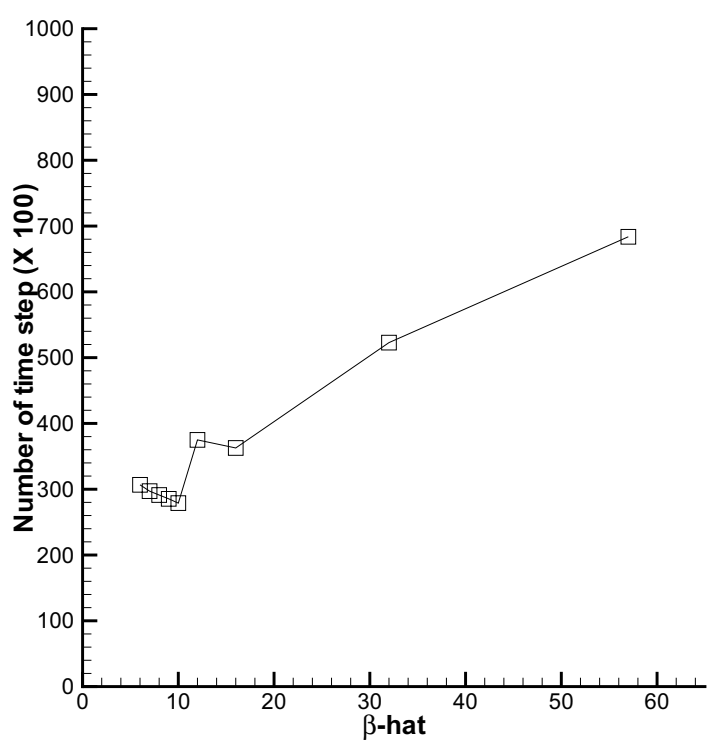

Figure 8 - Dependence on $\hat{\beta}$ of the number of time steps required to achieve convergence.
The dependence on the value of $\hat{\beta}$ of the number of iterations required to meet the convergence criterion LRHS $S_{\rho u} \leq 10^{-4}$ is presented in Fig. (8). The optimum value of $\hat{\beta}$, around 10 , obtained here confirmed the results reported by Bruel et al. (1996), and shows that such an optimum is poorly dependent on the numerical approach retained and thus can be considered as specific of the pseudo-compressibility approach itself.

\section{CONCLUDING REMARKS}

The proposed combination of the pseudocompressibility method with an unstructured formulation to solve the governing equations of a turbulent premixed flame proves to work properly and to produce results that compare well with those obtained in the literature on the very same flow geometry. Due to the centered type of spatial discretization used here, a fourth-order artificial numerical dissipation has been added to stabilize the calculations but the results show that the accuracy of the final solution has been preserved. The use of an explicit three-stage hybrid time stepping scheme shows that it is powerful enough to calculate the steady solution in the pseudo-time of the system of discretized governing equations. Nevertheless, the use of such an explicit time stepping scheme has the drawback of limiting the maximum possible CFL-number value resulting in an increased total number of pseudo-time steps required to achieve convergence. If needed, an implicit formulation could be considered to speed-up the convergence process. Finally, an auto-adaptive Lagrangian-Eulerian approach on unstructured grid (Trépanier et al., 1991; Nomura and Hughes, 1992) could greatly help in reducing the total number of grid elements required to resolve properly the high gradients present in the flow while preserving the overall accuracy of the calculations. Future work will focus on these directions.

\section{Acknowledgments}

The authors would like to acknowledge the financial support from CNPq, and CAPES. A large part of this work has been carried out during the stay of the first author at the Laboratoire de Combustion et de Détonique (Poitiers, France) that belongs to the Centre National de la Recherche Scientifique (CNRS) and in the framework of a "co-tutelle" thesis prepared under the co-supervision of the University of Poitiers and ITA. The authors further acknowledge the partial support of $\mathrm{CNPq}$ under the Integrated Project Research Grant N 522.413/96-0.

\section{REFERENCES}

Barth, T. J., and Jespersen, D. C, 1989, "The Design an Application of Upwind Schemes on Unstructured Meshes", $27^{\text {th }}$ AIAA Aerospace Sciences Meeting, AIAA Paper 89-0366.

Batina, J. T., 1989, "Vortex-Dominated ConicalFlow Computations using Unstructured Adaptively Refined 
Meshes", 20 ${ }^{\text {th }}$ Fluids Dynamics Conference, AIAA Paper 89-1816.

Bray, K. N. C., Champion, M., and Libby, P. A., 1984, "Mean Reaction Rates in Premixed Turbulent Flames", $22^{\text {nd }}$ Symposium (International) on Combustion, the Combustion Institute, pp. 763-769.

Bray, K. N. C., Libby, P. A., and Moss, J. B., 1984, "Flamelet Crossing Frequencies and Mean Reaction Rates in Premixed Turbulent Combustion", Combustion Science and Technology, Vol. 4, pp. 143-172.

Bray, K. N. C., and Moss, J. B., 1977, "A Unified Statistical Model of the Premixed Turbulent Flame", Acta Astronautica, Vol 4, pp. 291-319.

Bruel, P., Karmed, D., and Champion, M., 1996, "A Pseudo-Compressibility Method for Reactive Flows at Zero Mach Number", International Journal of Computational Fluid Dynamics, Vol. 7, pp. 291-310.

Chorin, A. J., 1967, "A Numerical Method for Solving Incompressible Viscous Flow Problems", Journal of Computational Physics, Vol. 2, pp. 12-26.

Corvellec, C., 1998, "Étude Numérique et Analytique des Caractéristiques Propagatives d'une Zone de Combustion se Développant au Sein d'un Écoulement Turbulent Prémélangée Modélisée par une Approche de Type Flammelette", Doctoral Thesis, University of Poitiers, France.

Corvellec, C., Bruel, P., and Sabelnikov, V. A., 1999, "A Time-Accurate Scheme for the Calculations of Unsteady Reactive Flows at Low Mach Number", International Journal for Numerical Methods in Fluids, Vol. 29, pp. 207-227.

Dourado, W. M. C., and Azevedo J. L. F., 1996, "Flow Simulation with Unstructured Meshes over Basic Automotive Configurations with All Speed Method", $6^{\text {th }}$ Brazilian Congress of Engineering and Thermal Sciences and $6^{\text {th }}$ Latin American of Mass and Heat Transfer, ENCIT/LATCYM 96, AMBC Editor, Vol. I, pp. 553-558. Florianópolis, Brazil.

Dourado, W. M. C., and Azevedo J. L. F., 1999, "Analysis of an All Speed Method in Laminar Flows using Unstructured Meshes", $15^{\text {th }}$ Brazilian Congress of Mechanical Engineering, ABCM Editor (CD-ROM), Águas de Lindóas, Brazil.

Dourado, W. M. C., Azevedo, J. L. F., and Bruel, P., 2000, "Application of the All Speed Method to Steady and Unsteady Flows on Unstructured Grids", 8 th Brazilian Congress of Thermal Engineering and Sciences, ABCM Editor (CD-ROM), Porto Alegre, Brazil.
Favre, A., 1965, "Équations des Gaz Turbulents Compressibles", Journal de Mécanique, Vol. 4, pp. 361391.

Frisch, U., "Turbulence", Cambridge University Press, 1995.

Jameson, A., Baker, T. J., and Weatherill, N.P., 1986, "Calculation of Inviscid Transsonic Flow over a Complete Aircraft", 24 ${ }^{\text {th }}$ AIAA Aerospace Sciences Meeting and Exhibit, AIAA Paper 86-0103.

Kolmogorov, A., Petrovskii, I., and Piscounov, N., 1937, "Study of the Diffusion Equation with Growth of the Quantity of Matter and its Application to a Biology Problem", Bulletin of The Moscow State University, International Serie, Vol. 1, Section A(6).

Launder, B. E., and Spalding, D. B., 1974, "The Numerical Computation of Turbulent Flows", Computer Methods in Applied Mechanics and Engineering, Vol. 3, pp. 269-289.

Majda, A. J. and Sethian, J., 1985, "The Derivation and Numerical Solution of the Equations for Zero Mach Number Combustion", Combustion Science and Technology, Vol. 42, pp. 185-205.

Manzari, M. T., Hassan, O., Morgan, K., and Weatherill, N. P., 1998, "Turbulent Flow Computation on 3D Unstructured Grids", Finite Elements in Analysis and Design, Vol. 30, pp. 353-363.

Mavriplis, D. J., 1998, "Multigrid Strategies for Viscous Flow Solvers on Anisotropic Unstructured Meshes", ICASE Report 98-6.

Mavriplis, D. J., Jameson, A., and. Martinelli, L., 1989, "Multigrid Solution of the Navier-Stokes equations on Triangular Meshes", ICASE, NASA CR- 89-11.

Mavriplis and Venkatakrishnan, V., 1995, "A Unified Multigrid Solver for the Navier-Stokes Equations on Mixed Element Meshes", ICASE Report 95-53.

Nomura, T., and Hughes, T. J. R., 1992, "An Arbitrary Lagrangian-Eulerian Finite Element Method for Interaction of Fluid and Rigid Body", Computer Methods in Applied Mechanics and Engineering, Vol. 95, pp. 115-138.

Trépanier, J. Y., Reggio, M., Zhang, H., and Camarero, R., 1991, "A Finite-Volume Method for the Euler Equations on Arbitrary Lagrangian-Eulerian Grids", Computer and Fluids, Vol. 20, No. 4, pp. 399-409. 